OPEN ACCESS

Edited by:

Akio Adachi,

Tokushima University, Japan

Reviewed by:

Martin Skarzynski,

National Cancer Institute,

United States

Tohru Kiyono,

National Cancer Center Research

Institute, Japan

${ }^{*}$ Correspondence:

Jie Cui

jiecui@wh.iov.cn

Mingyue Chen

chmy@wh.iov.cn

tThese authors have contributed equally to this work.

Specialty section: This article was submitted to Virology,

a section of the journal

Frontiers in Microbiology

Received: 21 June 2017

Accepted: 24 August 2017

Published: 19 September 2017

Citation:

Wang $H$, Zhao $Y$, Chen M and Cui J

(2017) Identification of Novel Long

Non-coding and Circular RNAs in Human Papillomavirus-Mediated

Cervical Cancer.

Front. Microbiol. 8:1720.

doi: 10.3389/fmicb.2017.01720

\section{Identification of Novel Long Non-coding and Circular RNAs in Human Papillomavirus-Mediated Cervical Cancer}

\author{
Hongbo Wang ${ }^{1 \dagger}$, Yingchao Zhao ${ }^{2 \dagger}$, Mingyue Chen ${ }^{3 *}$ and Jie Cui ${ }^{3 *}$ \\ ' Department of Obstetrics and Gynecology, Union Hospital, Tongji Medical College, Huazhong University of Science and \\ Technology, Wuhan, China, ${ }^{2}$ Cancer Center, Union Hospital, Tongji Medical College, Huazhong University of Science and \\ Technology, Wuhan, China, ${ }^{3}$ CAS Key Laboratory of Special Pathogens and Biosafety, Center for Emerging Infectious \\ Diseases, Wuhan Institute of Virology, Chinese Academy of Sciences, Wuhan, China
}

Cervical cancer is the third most common cancer worldwide and the fourth leading cause of cancer-associated mortality in women. Accumulating evidence indicates that long non-coding RNAs (IncRNAs) and circular RNAs (circRNAs) may play key roles in the carcinogenesis of different cancers; however, little is known about the mechanisms of IncRNAs and circRNAs in the progression and metastasis of cervical cancer. In this study, we explored the expression profiles of IncRNAs, circRNAs, miRNAs, and mRNAs in HPV16 (human papillomavirus genotype 16) mediated cervical squamous cell carcinoma and matched adjacent non-tumor (ATN) tissues from three patients with high-throughput RNA sequencing (RNA-seq). In total, we identified 19 IncRNAs, 99 circRNAs, 28 miRNAs, and 304 mRNAs that were commonly differentially expressed (DE) in different patients. Among the non-coding RNAs, 3 IncRNAs and 44 circRNAs are novel to our knowledge. Functional enrichment analysis showed that DE IncRNAs, miRNAs, and mRNAs were enriched in pathways crucial to cancer as well as other gene ontology (GO) terms. Furthermore, the co-expression network and function prediction suggested that all $19 \mathrm{DE}$ IncRNAs could play different roles in the carcinogenesis and development of cervical cancer. The competing endogenous RNA (ceRNA) network based on DE coding and non-coding RNAs showed that each miRNA targeted a number of IncRNAs and circRNAs. The link between part of the miRNAs in the network and cervical cancer has been validated in previous studies, and these miRNAs targeted the majority of the novel non-coding RNAs, thus suggesting that these novel non-coding RNAs may be involved in cervical cancer. Taken together, our study shows that DE noncoding RNAs could be further developed as diagnostic and therapeutic biomarkers of cervical cancer. The complex ceRNA network also lays the foundation for future research of the roles of coding and non-coding RNAs in cervical cancer.

Keywords: cervical cancer, RNA-seq, IncRNAs, circRNAs, ceRNA, HPV 


\section{INTRODUCTION}

Cervical cancer is the third most common type of cancer and the fourth leading cause of cancer-associated mortality in women, with an estimated 526,000 new cases and 239,000 deaths in 2015 globally (Fitzmaurice et al., 2017). Cervical cancer is caused by a persistent infection with high-risk human papillomavirus (HPV), including HPV-16, HPV-18, and HPV-53. HPV16 and HPV18, the most frequently detected subtypes worldwide, contribute to $70 \%$ of all invasive cervical cancer cases (de Sanjose et al., 2010). The carcinogenesis of HPV is dependent on the activities of viral oncoproteins E5, E6, and E7 (McLaughlin-Drubin et al., 2012). However, in most cases, women infected by HPV remain asymptomatic because an adequate immune response is capable of controlling the infection, and very few cases progress to cervical cancer (Insinga et al., 2011). Previous studies have suggested that abnormal host genes as well as additional factors, such as multiparity and smoking, influence the risk of cervical cancer development (Vaccarella et al., 2006; Louie et al., 2011; de Freitas et al., 2014). Therefore, the discovery of vital diagnostic and therapeutic molecular markers could offer substantial help in controlling the infection of HPV and preventing the progression of cervical cancer.

In recent years, much attention has been paid to two new classes of RNA, long non-coding RNAs (lncRNAs) and circular RNAs (circRNAs). LncRNAs are non-protein coding transcripts that are longer than 200 nucleotides (Cao, 2014). The newly identified non-coding circRNAs are single-stranded, covalently closed circular molecules. Both can act as microRNA sponges and regulate gene expression by functioning as competing endogenous RNA (ceRNA) in distinct physiological and pathophysiological states (Li et al., 2017). Mounting evidence shows lncRNAs and circRNAs play key roles in diseases, especially in cancer, and can serve as diagnostic or predictive biomarkers (Qu et al., 2015). However, as far as we know, no research has been completed about the function of circRNAs in cervical cancer. Furthermore, the entire ceRNA network related to cervical cancer is unclear.

In this study, we performed high-throughput RNA sequencing (RNA-seq) of cervical squamous cell carcinoma (CSCC) and matched adjacent non-tumor (ATN) tissues from three patients. First, we identified differentially expressed (DE) lncRNAs, circRNAs, miRNAs, and mRNAs between CSCC and ATN tissues. More importantly, three lncRNAs and 44 circRNAs have not been reported before. Second, gene ontology (GO) and Kyoto encyclopedia of genes and genomes (KEGG) pathway enrichment analyses were performed. Additionally, co-expression and function analyses were carried out to reveal the potential biological roles of the lncRNAs. Finally, we constructed a ceRNA network of coding and non-coding RNAs to predict the interaction among lncRNAs, circRNAs, miRNAs, and mRNAs. We found that the majority of the novel non-coding RNAs may be involved in cervical cancer. Our findings revealed for the first time that circRNAs are likely to be involved in cervical cancer and DE non-coding RNAs could be further developed as biomarkers. The complex ceRNA network might help to understand the interplay among coding and non-coding
RNAs in the carcinogenesis and the proliferation of cervical cancer.

\section{MATERIALS AND METHODS}

\section{Tissue Samples}

Three pairs of freshly frozen tissues of CSCC and matched adjacent non-tumor tissue (ANT) were collected from three cervical cancer stage IB1 patients (caused by HPV16) who underwent a radical hysterectomy between June 2016 and December 2016 at Union Hospital, Tongji Medical College, Huazhong University of Science and Technology. The collected adjacent normal tissues were $2 \mathrm{~cm}$ away from the visible cervical cancer lesions. Once the tissues were dissected, they were immersed in RNAlater (Qiagen $\mathrm{GmbH}$, Hilden, Germany) for $30 \mathrm{~min}$, and they were then stored in liquid nitrogen until further use. Cervical cancer diagnosis was based on the International Federation of Gynecology and Obstetrics (FIGO) criteria. The diagnosis of all of the cases was histologically confirmed by two independent pathologists, and all of the tumor tissues were assessed by Hematoxylin and Eosin (HE) staining. The procedures were approved by the ethics committee of Union Hospital, Tongji Medical College, and informed consent was obtained from all of the patients.

\section{RNA Extraction and Quality Control}

Total RNA was isolated using TRIzol reagent (Invitrogen, Carlsbad, CA, United States) according to the manufacturer's protocol. The concentration and integrity of the total RNA were measured by the Qubit ${ }^{\circledR}$ RNA Assay Kit in Qubit ${ }^{\circledR} 2.0$ Flurometer (Life Technologies, Foster City, CA, United States) and RNA Nano 6000 Assay Kit of the Bioanalyzer 2100 system (Agilent Technologies, Santa Clara, CA, United States).

\section{RNA-seq and Data Analysis}

Three biological replicates of the CSCC and control samples were used for lncRNA, circRNA, and mRNA sequencing, while three biological replicates of the CSCC and control samples were mixed respectively for miRNA sequencing. Sequencing libraries of lncRNAs, circRNAs, and mRNAs were generated using the rRNA-depleted RNA by NEBNext Ultra Directional RNA Library Prep Kit for Illumina (New England Biolabs, Ipswich, MA, United States) following manufacturer's recommendations, and the sequencing libraries of miRNA were generated using Small RNA Sample Pre Kit (Illumina, San Diego, CA, United States). RNA-seq sequencing was performed using an Illumina HiSeq 2500 (Illumina, San Diego, CA, United States). Raw data (raw reads) of the fastq format were first processed using FASTX-Toolkit $(\mathrm{v} 0.0 .13)^{1}$. In this step, clean data (clean reads) were obtained by removing reads containing an adapter, reads containing poly- $\mathrm{N}$, and low-quality reads from raw data. At the same time, Q20, Q30, and GC content of the clean data were calculated. Paired-end reads were aligned to

\footnotetext{
${ }^{1}$ http://hannonlab.cshl.edu/fastx_toolkit/index.html
} 
the human genome with Tophat (v 2.0.9) (Langmead et al., 2009). Reads that were mapped to the human genome were assembled using Cufflinks (v 2.1.1) (Trapnell et al., 2010). The miRNA reads were aligned to known mature human microRNA sequences using Bowtie (v 2.0.6) (Langmead et al., 2009). Cuffdiff (v 2.1.1) was used to calculate the FPKMs (expected number of Fragments Per Kilobase of transcript sequence per Million base pairs sequenced) of both lncRNAs and protein-coding genes in each sample (Trapnell et al., 2012). Transcripts with $P$-values $<0.05$ were assigned as DE. TPM normalization is used for estimating relative circRNAs and miRNAs production levels from RNA-seq data (Zhou et al., 2010). Differential expression of circRNAs was determined with DEseq2 (Love et al., 2014), and a $P$-value $<0.05$ was considered to be a threshold to measure differential expression. DE miRNAs were identified using DEGseq (Wang et al., 2010), with the Q value $<0.01$ and $\mid \log 2$ (foldchange) $\mid>1$ used as the threshold to evaluate the statistical significance of the miRNA expression differences. The whole analysis workflow was shared on Galaxy platform ${ }^{2}$ and available at https://usegalaxy.org/u/chmy/w/rna-seq-differentialanalysis.

\section{Functional Enrichment Analysis}

Gene ontology analysis ${ }^{3}$ was conducted to annotate the genes with terms under the biological process, cellular component, and molecular function categories using the DAVID bioinformatics tool (Huang da et al., 2009). KEGG enrichment analysis was also performed to predict the molecular interactions and reaction networks with KOBAS software using a hypergeometric test (Xie et al., 2011). GO terms and KEGG pathways with $P$-values $<0.05$ were regarded as being significantly enriched. The $-\log 10(P$-value) denotes the enrichment score representing the significance of the corresponding GO term and pathway enrichment among DE genes.

\section{Correlation and Co-expression Analysis}

Co-expression analysis was conducted by calculating the Pearson correlation coefficient (PCC) between the lncRNAs and the known protein-coding genes according to their expression levels. The selection parameter $|\mathrm{PCC}|>0.95$ was set as the threshold in the co-expression analysis. The co-expression network was illustrated using Cytoscape (Shannon et al., 2003).

\section{Competing Endogenous RNA (ceRNA) Network Analysis}

The potential miRNA response elements (MREs) of lncRNA, circRNAs, and mRNAs were searched using miRanda ${ }^{4}$. The overlapping of the same miRNA seed sequence binding site on both the lncRNA/circRNA and the mRNA was considered to be a potential lncRNA/circRNA-miRNA-mRNA interaction. The ceRNA network was illustrated using Cytoscape.

\footnotetext{
${ }^{2}$ http://www.usegalaxy.org

${ }^{3}$ http://www.geneontology.org

${ }^{4}$ http://www.microrna.org/microrna/home.do
}

\section{RESULTS}

\section{Identification of DE IncRNAs, circRNAs, miRNAs, and mRNAs}

Summary of total RNA-seq data from CSCC and ATN tissues are listed in Supplementary Table S1, which demonstrated the relatively high quality of the transcriptome data. To date, there has been no report on circRNAs in cervical cancer patients. Here, for the first time, we reveal the circRNAs related to cervical cancer. RNA-seq analysis showed thousands of lncRNAs, circRNAs, miRNAs, and mRNAs expressed in cervical cancer patients. We compared the expression profiles of lncRNAs, circRNAs, miRNAs, and mRNAs between CSCC and ANT tissues from three patients, and found 19 lncRNAs, 99 circRNAs, 28 miRNAs, and 304 mRNAs were DE (Figure 1A). Among them, 11 lncRNAs, 58 circRNAs, 15 miRNAs, and 158 mRNAs were upregulated, and 8 lncRNAs, 41 circRNAs, 13 miRNAs, and 146 mRNAs were downregulated in the CSCC samples compared with the controls. DE non-coding RNAs are listed in Supplementary Table S2.

The DE circRNAs and mRNAs were widely scattered throughout the autosomal chromosomes, while lncRNAs and miRNAs were only distributed in 13 and 12 chromosomes, respectively (Figure 1B). According to their location relative to nearby protein-coding genes, lncRNAs could be classified into five categories: sense lncRNAs, antisense lncRNAs, intronic lncRNAs, intergenic lncRNAs, and bidirectional lncRNAs (Ponting et al., 2009). Among 19 DE lncRNAs, 14 lncRNAs (73.7\%) were intergenic. It has been suggested that circRNAs are mainly generated from exons (exonic circRNA, ecircRNA), introns (intronic circRNA, ciRNA), or both (exon-intron circRNA, EIciRNA) (Meng et al., 2016). In this study, 64 (64.6\%) DE circRNAs were EIciRNAs, and only 4 (4.0\%) were ecircRNAs.

\section{Novel IncRNAs and circRNAs}

Among the DE coding and non-coding RNAs, we uncovered 3 novel lncRNAs (LNC_000188, LNC_000226, and LNC_000231) and $44(44.4 \%)$ novel circRNAs that have not been reported before (Supplementary Table S2). LNC_000226 and LNC_000231 were distributed in chromosome 5, and presented different trends of expression with LNC_000226 downregulated and LNC_000231 upregulated. LNC_000188 was located in chromosome 3 and upregulated. Novel circRNAs were widely scattered in 17 chromosomes. Interestingly, 25\% were distributed in chromosome 1 . Twenty-three (52.3\%) novel circRNAs were upregulated. EIciRNAs also constitute the majority $(70.5 \%)$ of the novel circRNAs.

\section{GO and KEGG Pathway Analyses}

To predict the potential functional implication of the DE non-coding RNAs as well as mRNAs, we performed GO and KEGG functional enrichment analyses. To some extent, the function of lncRNAs could be inferred through their associated mRNAs by cis-regulation and trans-regulation (Sun et al., 2013). It is well-known that cis-acting lncRNAs target neighboring genes (Ponjavic et al., 2009; Orom et al., 2010); therefore, 

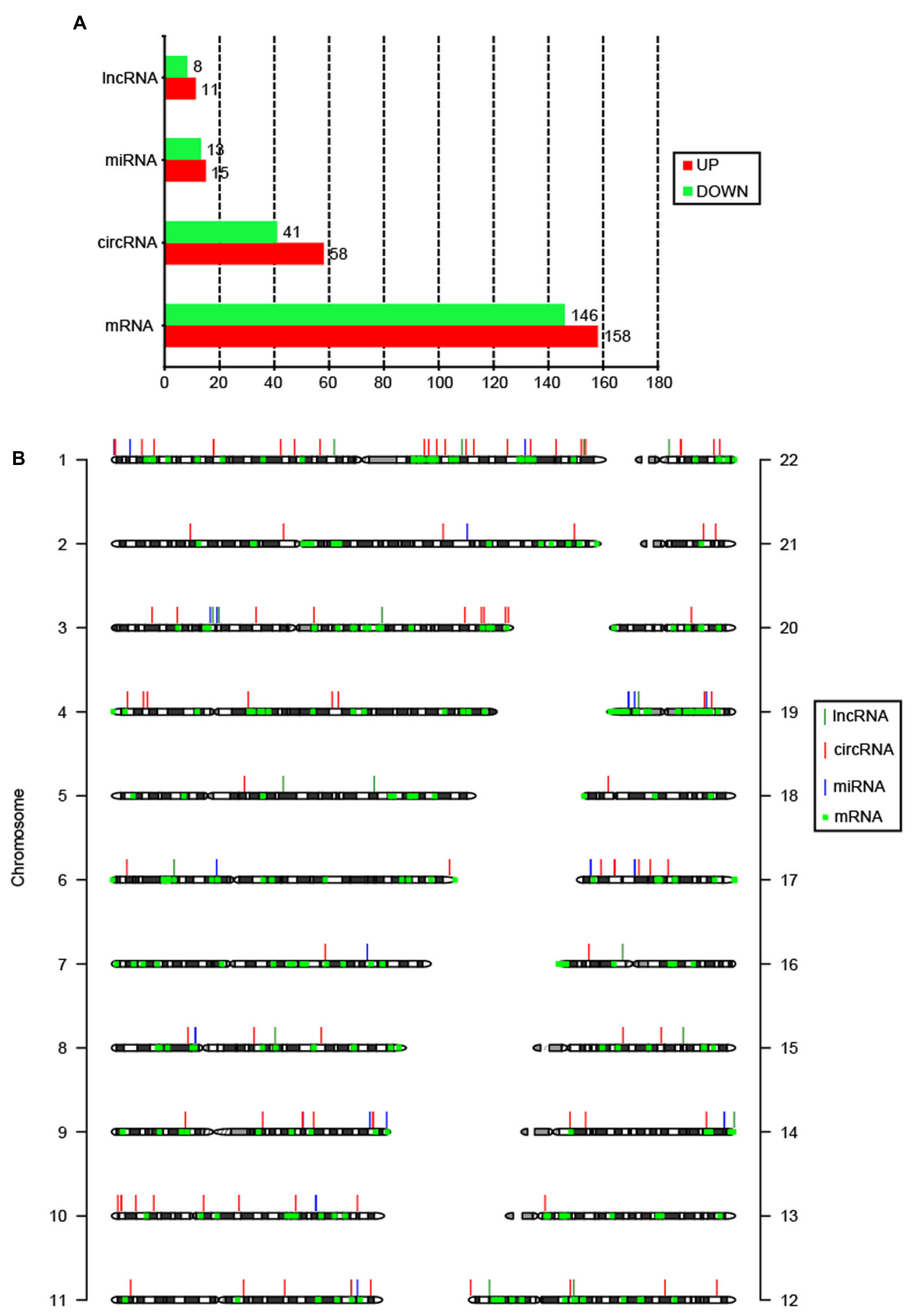

FIGURE 1 | Features of DE coding and non-coding RNAs. (A) Number of upregulated and downregulated IncRNAs, circRNAs, miRNAs, and mRNAs. (B) Chromosomal distribution of DE IncRNAs, circRNAs, miRNAs, and mRNAs.

protein-coding genes $100-\mathrm{kb}$ upstream and downstream of all of the DE lncRNAs were searched. We also predicted the potential targets of lncRNAs in trans-regulatory relationships using co-expression analysis, and the union of mRNAs resulting from cis-regulation and trans-regulation was used to conduct functional enrichment analysis. Our data showed that for DE lncRNAs, the most relevant Go terms associated with biological process included nuclear-transcribed mRNA catabolic process, 
SRP-dependent cotranslational protein targeting to membrane, viral transcription, translational initiation, and cell-cell adhesion (Figure 2A). Crucially, viral transcription and cell-cell adhesion play pivotal roles in the development and progression of cancer (Okegawa et al., 2004; Schelhorn et al., 2013). KEGG pathway analysis suggested that the most frequently predicted pathways were involved in metabolism, Huntington's disease, and oxidative phosphorylation, Ribosome and Alzheimer's disease, while pathways, including viral carcinogenesis and pathways in cancer, were also enriched with a relatively low enrichment score (Supplementary Figure S1A). These results indicate that DE lncRNAs may participate in the formation and development of cervical cancer.

Up to now, most circRNAs have not been functionally annotated (Salzman, 2016). To explore the potential function of DE circRNAs, GO and pathway analyses of circRNA hosting genes were performed. Our data suggested that only one GO term related to biological process was enriched, and it was involved in endosomal vesicle fusion (Figure 2B). The KEGG pathways only included nicotinate and nicotinamide metabolism and lysine degradation.

The function of DE miRNAs was also investigated. The most enriched GO terms related to biological process included insulin receptor signaling pathway, G1/S transition of mitotic cell cycle, DNA replication, transport and response to drug (Figure 2C). What is remarkable is that the top-two enriched pathways were proteoglycans in cancer and pathways in cancer (Supplementary Figure S1B). Therefore, it is also possible that DE miRNAs may be important in the pathophysiology of cervical cancer.

For DE mRNAs, strong GO enrichment focusing on biological processes was observed in nucleosome assembly, DNA replication-dependent nucleosome assembly, telomere organization, chromatin silencing at rDNA, and the positive regulation of gene expression, epigenetic (Figure 2D). KEGG pathway analysis showed that mRNAs were significantly enriched in the pathways involved in systemic lupus erythematosus, alcoholism, oxidative phosphorylation, non-alcoholic fatty liver disease, and viral carcinogenesis (Supplementary Figure S1C), which is directly correlated with tumorigenesis.

\section{Co-expression Analysis of IncRNAs/mRNAs and Function Prediction of IncRNAs}

We constructed a coding-non-coding gene (CNC) co-expression network based on the correlation analysis between $19 \mathrm{DE}$ lncRNAs and 304 DE mRNAs. Eventually, we found 230 mRNAs (127 upregulated and 103 downregulated) that had a strong correlation with 19 lncRNAs (11 upregulated and 8 downregulated) according to the PCC analysis (Figure 3).

Furthermore, to reveal the potential function of key lncRNAs, we conducted GO and KEGG pathway analysis based on the target genes. In the KEGG pathway analysis, we only presented the top-five enriched pathways of lncRNAs, which can be classified into six categories (cancers, immune system, signal transduction, cellular community - eukaryotes, other diseases, and other metabolic pathways). It is worthy to note that 11 lncRNAs (LNC_000231, LNC_000226, ENST00000 630242.2, ENST00000426585.5, ENST00000426185.1, ENST000 00471754, ENST00000518880.1, ENST00000429269.1, ENST000 00562869.1, ENST00000566942.1, and ENST00000549830.2) participated in at least one pathway related to cancer, and some lncRNAs may also be involved in immune system, signal transduction, and cellular community, including adherens junction (Figure 4), all of which may play a role in the tumorigenesis and progression of cancer. Although lncRNA ENST00000454467.5 has not been enriched in the pathway related to cancer, it was significantly enriched in the pathway involved in platinum drug resistance that is related to cervical cancer cell proliferation and apoptosis (Wang et al., 2017). The remaining seven lncRNAs (ENST00000 482019.1, ENST00000421735.1, LNC_000188, ENST00000 397381.4, ENST00000539009.1, ENST00000566260.1, and ENST00000553843.5) possibly interacted with mRNAs in the KEGG pathways, including Huntington's disease, Alzheimer's disease, ribosome, oxidative phosphorylation, and metabolic pathways, and these pathways did not seem to be implicated in cancer. However, GO analysis suggested that six lncRNAs were enriched in the GO term named viral transcription, except for lncRNA ENST00000482019.1, which may be involved in cell adhesion and signal transduction according to the GO analysis (Supplementary Table S3). Moreover, a previous study suggested that IncRNA UCA1 (ENST00000397381.4) could play an important role in the pathogenesis of cervical cancer (Wang et al., 2017). Therefore, 19 DE lncRNAs could play different roles in the tumorigenesis and development of cervical cancer, and they might even function as tumor suppressors or oncogenes. Also, the lncRNAs may represent promising potential biomarkers of cervical cancer, but further work is needed to elucidate the detailed mechanisms of the lncRNAs.

\section{Construction of the ceRNA Network}

Recent studies have shown that within the ceRNA network, both lncRNAs and circRNAs can interact with miRNAs through microRNA response elements (MREs) (Salmena et al., 2011; Hansen et al., 2013). We constructed three comprehensive ceRNA networks that integrated the expression profiles and regulatory relationships of DE lncRNAs, circRNAs, miRNAs, and mRNAs from our RNA-seq data. We detected 19 miRNA-mediated IncRNA-mRNA competing triplets among 19 miRNAs, 15 lncRNAs, and 60 mRNAs (Figure 5A); 27 miRNA-mediated circRNA-mRNA competing triplets among 27 miRNAs, 80 circRNAs, and 62 mRNAs (Supplementary Figure S2); and 19 miRNA-mediated lncRNA-circRNAmRNA competing quaternities among 19 miRNAs, 15 lncRNAs, 74 circRNAs, and 61 mRNAs (Supplementary Figure S3). Several of the miRNAs have been linked to cervical cancer, especially hsa-miR-1246, hsa-miR-486-3p, hsa-miR-425-5p, hsa-miR-206, hsa-miR-135b-5p, hsa-miR150-5p, hsa-miR-195-5p, and hsa-miR-497-5p (Luo et al., 2013; Chen et al., 2014; Li et al., 2015; Ling et al., 2015; $\mathrm{Xu}$ et al., 2015; Ye et al., 2016; Shen et al., 2017; Sun et al., 2017), all of which have been implicated in the carcinogenesis and development of cervical cancer. Two 


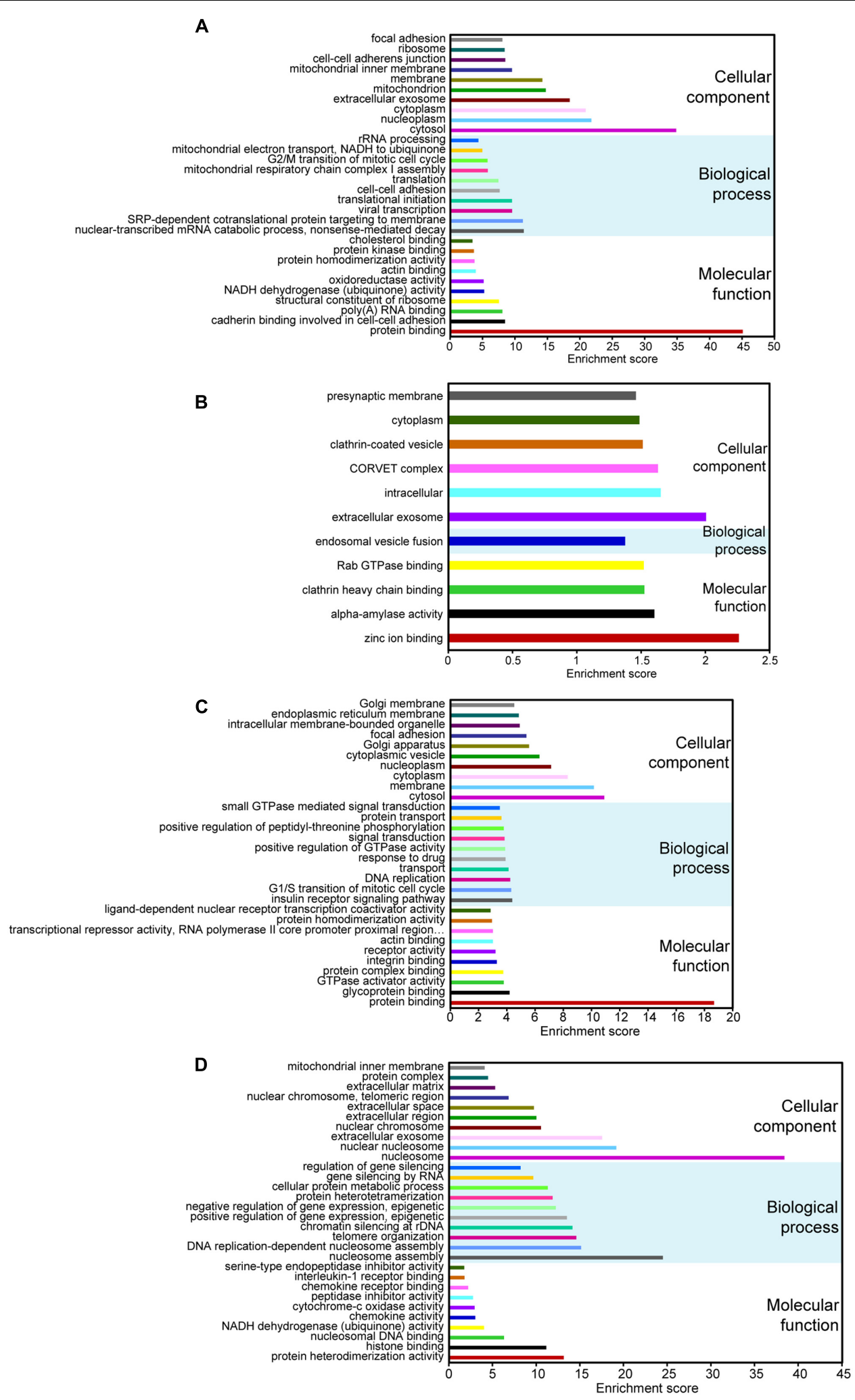

FIGURE 2 | Gene ontology (GO) analyses of DE coding and non-coding RNAs. GO annotations of DE IncRNAs (A), circRNAs (B), miRNAs (C), and mRNAs (D) with the top-ten enrichment score covering domains of biological process, cellular components, and molecular function. CircRNAs with less than $30 \mathrm{GO}$ terms were enriched. 


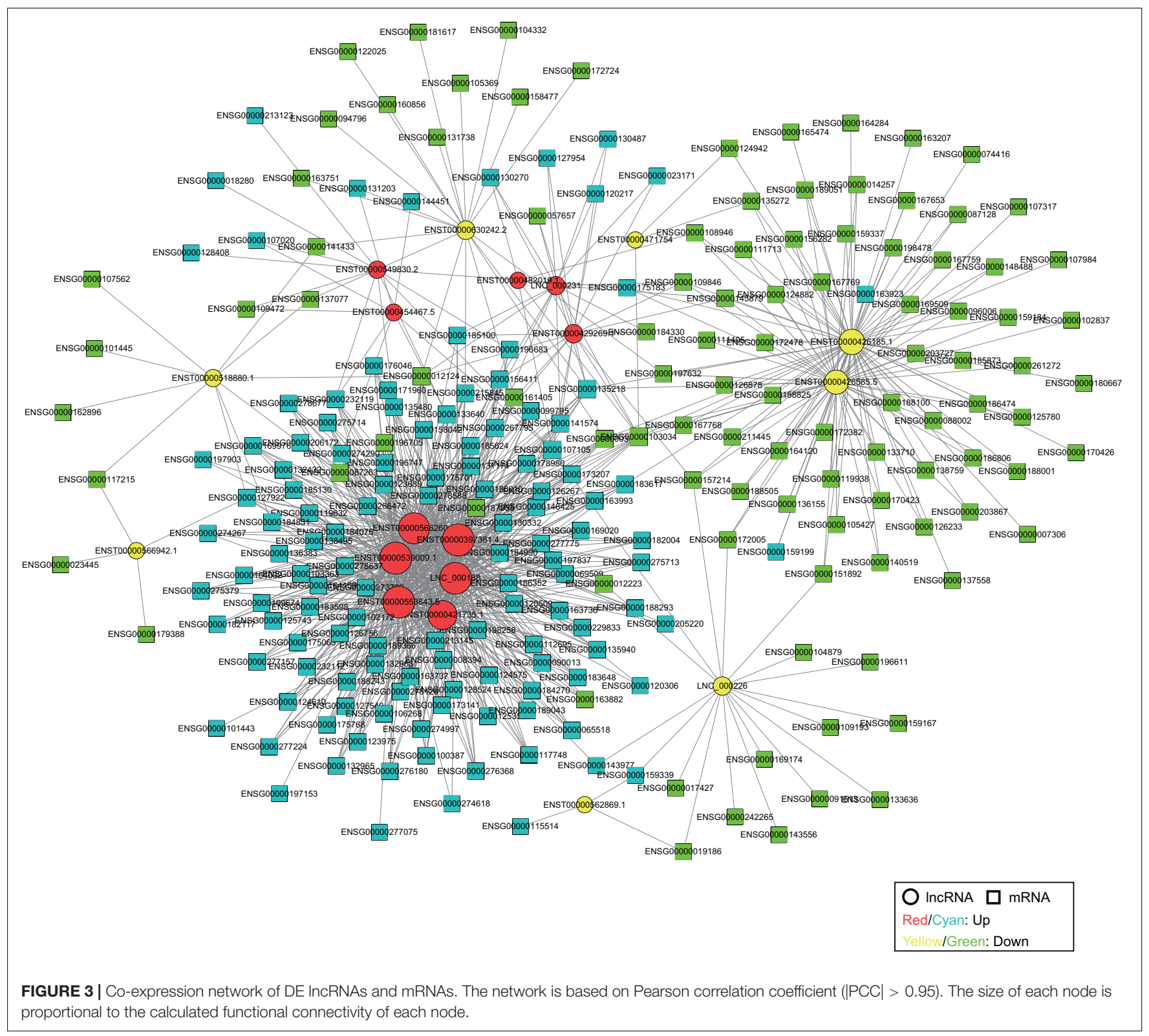

novel lncRNAs and 28 novel circRNAs were targeted by these miRNAs, and they accounted for the majority of the novel identified non-coding RNAs, thus implying their potential function in cervical cancer. For example, hsa-miR-425-5p is upregulated in cervical cancer tissue and suggested as a potential prognostic biomarker for cervical cancer (Sun et al., 2017), but the underlying molecular mechanisms are still unclear. The potential interaction was revealed through the ceRNA network, showing that hsa-miR-425$5 p$ targeted lncRNA HCG22 (ENST00000426185.1) and 15 circRNAs, including 5 novel circRNAs (hg38_circ_0008995, hg38_circ_0010744, hg38_circ_0011797, hg38_circ_0014951, and hg38_circ_0021570), thus enhancing our understanding of the interactions within IncRNA, circRNA, miRNA, and mRNA, especially novel lncRNA and circRNA.
LncRNA UCA1 (ENST00000397381.4) is the star biomarker in various types of cancers, such as breast cancer, gastric cancer, and lung cancer (Lee et al., 2016; Nie et al., 2016; Shang et al., 2016). Moreover, it has been proven that UCA1 is associated with cisplatin resistance, and it can be used as a potential target for a novel therapeutic strategy for cervical cancer (Wang et al., 2017). In this study, UCA1 was upregulated and interacted with hsa-miR-206, hsa-miR-3158-3p, hsa-miR3158-5p, hsa-miR-486-3p, hsa-miR-135a-5p and hsa-miR-135b$5 p$ (Supplementary Figure S3). These miRNAs also target a number of lncRNAs, cirRNAs, and mRNAs, including novel identified non-coding RNAs, thus providing potential evidence to reveal the correlation with tumor progression. Two novel lncRNAs (LNC_000188 and LNC_000231) were also included in the ceRNA network (Figure 5 and Supplementary Figure S3). 


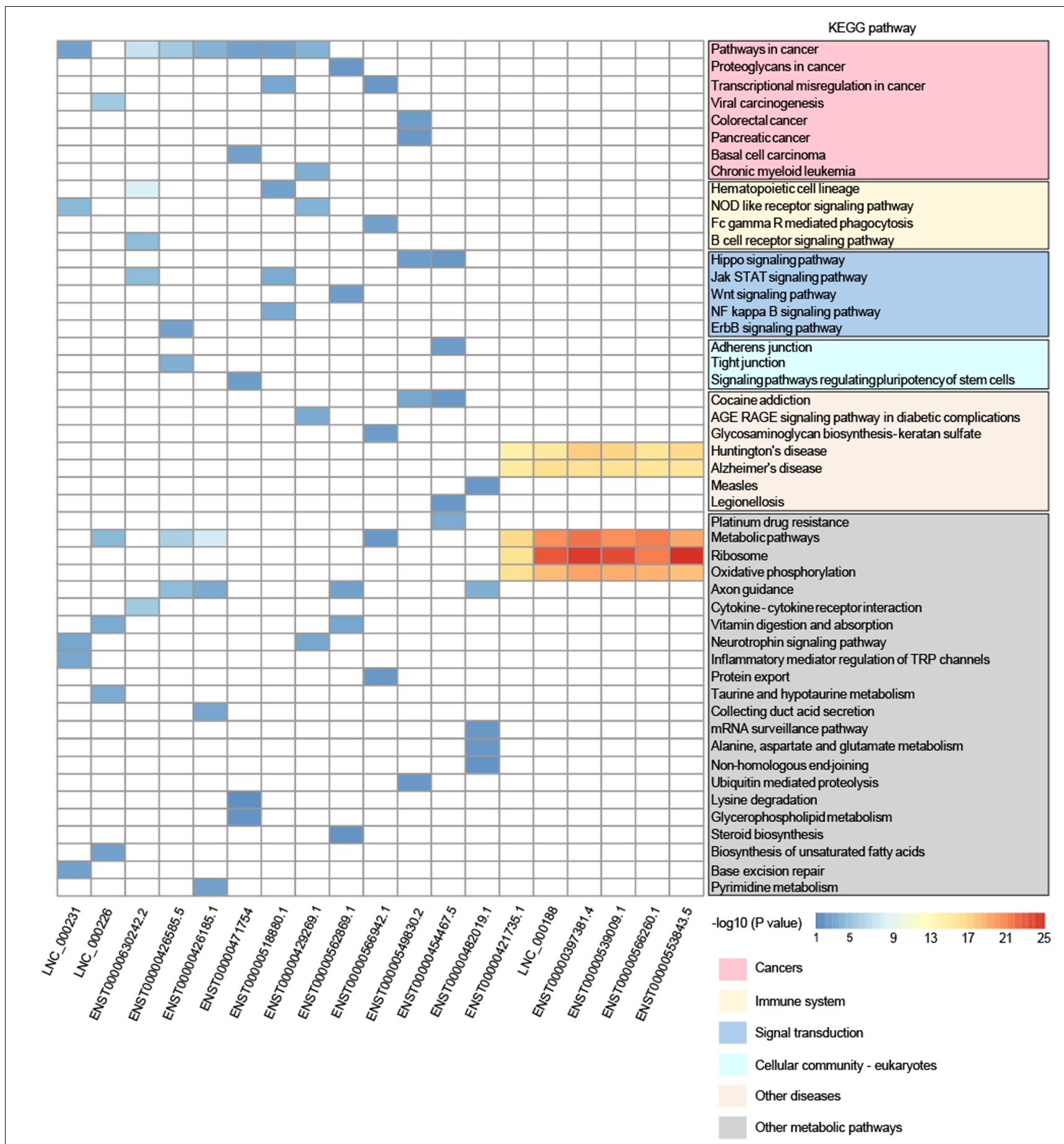

FIGURE 4 | Comparison of KEGG pathway enrichment analysis of DE IncRNAs based on the target genes. Only pathways within the top-five enrichment scores are presented. The pathways could be classified into six categories: cancers, immune system, signal transduction, cellular community - eukaryotes, other diseases, and other metabolic pathways.

The ceRNA network presented that lncRNAs LNC_000188 and LNC_000231 interacted with 4 (hsa-miR-195-5p, hsamiR-486-3p, hsa-miR-206, and hsa-miR-497-5p) and 6 (hsamiR-144-3p, hsa-miR-1246, hsa-miR-497-5p, hsa-miR-195-5p, hsa-miR-27b-3p, and hsa-miR-3158-5p) miRNAs, respectively
(Figures 5B,C). All of the miRNAs targeted by LNC_000188 and 3 of the miRNAs targeted by LNC_000231 were involved in the carcinogenesis and proliferation of cervical cancer (Luo et al., 2013; Chen et al., 2014; Ling et al., 2015; Ye et al., 2016; Shen et al., 2017); this may explain the 


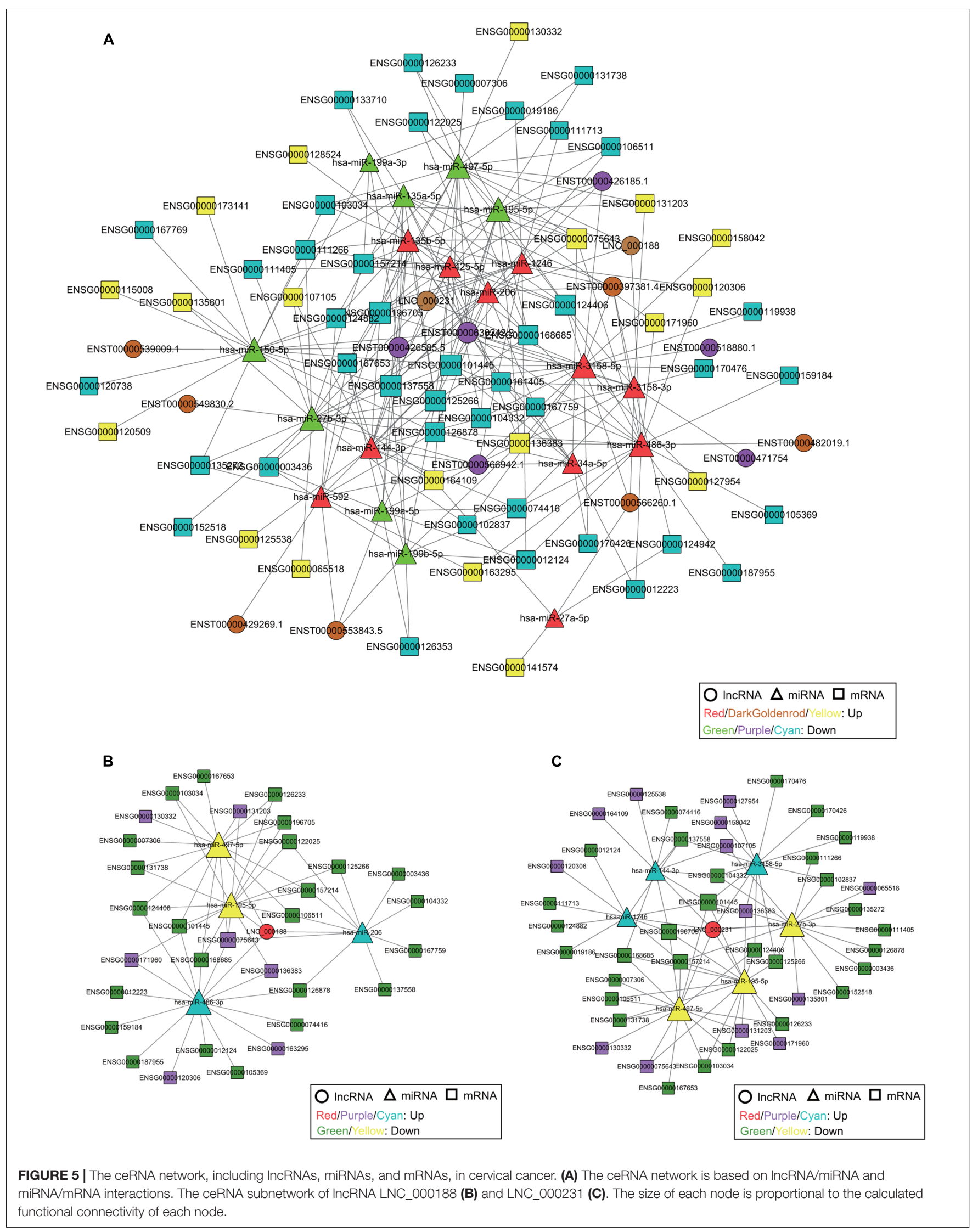


potential function related to cervical cancer of the two novel lncRNAs.

\section{DISCUSSION}

Emerging evidence shows that both IncRNAs and circRNAs play important roles in diseases, particularly cancer (Hauptman and Glavac, 2013; Wang et al., 2016; Dong et al., 2017). Recent advancements in surveying lncRNAs and circRNAs revealed that both can be used as potential diagnostic or predictive biomarkers for diseases, particularly for cancer development, progression, and prognosis. However, a comprehensive analysis of DE profiles of lncRNAs and circRNAs in cervical cancer has not been reported until now. To explore the functions and complex interactions of non-coding RNAs, we characterized the extensive transcription landscape related to the genesis and progression of cervical cancer.

We finally identified 19 lncRNAs, 99 circRNAs, 28 miRNAs, and 304 mRNAs that were DE between CSCC and ATN tissues using RNA-seq. To our knowledge, this is the first report of circRNA related to cervical cancer. Both IncRNAs and miRNAs were located in about half chromosomes whereas circRNAs and mRNAs were found throughout the autosomal chromosomes. The majority of the IncRNAs were intergenic, and most circRNAs were EIciRNAs. We also identified 3 novel lncRNAs and 44 novel circRNAs.

In order to explore the potential functions of the $\mathrm{DE}$ transcripts, bioinformatics methods, including GO and KEGG pathway analysis, were carried out in the study. GO analysis of IncRNAs showed that under the biological process some terms, including viral transcription and cell-cell adhesion, were enriched. Moreover, KEGG pathway analysis suggested that the pathways in cancer were also enriched. Likewise, KEGG pathway analysis of miRNAs and mRNAs revealed several pathways that could have important roles in the tumorigenesis mechanisms of cancer. This indicated that DE lncRNAs, miRNAs, and mRNAs could be correlated with cervical cancer. What was unexpected was that GO and KEGG analyses of the circRNA hosting genes only found a few enriched GO terms and pathways, all of which did not seem to be associated with cancer. One of the possibilities is that the hosting genes may be not suitable to analyze the function of circRNAs. Thus, we also used the ceRNA network to infer the biological role of circRNAs in this study.

In particular, to further reveal the function of lncRNAs, we constructed a co-expression network based on the correlation analysis between DE lncRNAs and mRNAs. There were 230 mRNAs (127 upregulated and 103 downregulated) and 19 lncRNAs (11 upregulated and 8 downregulated) that were presented in the co-expression network, and several of them have been found to be involved in cancer in previous studies. For example, Dickkopf1 (DKK-1, ENSG00000107984) acts as a new biomarker in human breast cancer (Liu J.T. et al., 2017), and it also exhibits transcriptional repression by epigenetic inactivation in specific cervical cancer cell lines, thus it may contribute to the constitutive activation of the Wnt signaling pathway in cervical carcinogenesis (Lee et al., 2008). A large number of studies have demonstrated that chemokines are involved in the progression, migration, and survival of cancers, such as CXCL12 (ENSG00000107562), CCL21 (ENSG00000137077), and especially CCL19 (ENSG00000172724), which is associated with progression of cervical cancer (Teicher and Fricker, 2010; Xiong et al., 2017; Zhang et al., 2017). Homeobox B13 (HOXB13, ENSG00000159184) is a susceptibility gene for prostate cancer (Xu et al., 2013). The co-expression network showed the interaction between lncRNAs and mRNAs, for example, CCL21 interacted with lncRNA KIAA0125 (ENST00000630242.2) and RP11-363E6.3 (ENST00000518880.1), and HOXB13 interacted with IncRNA HCG22 (ENST00000426185.1) and TPTEP1 (ENST00000426585.5). Thus, a co-expression network provides a better understanding of the biological functions of lncRNAs and mRNAs in cervical cancer. GO and KEGG pathway analyses suggested that $19 \mathrm{DE}$ lncRNAs could play different roles in cancer development. Therefore, the lncRNAs may represent promising potential biomarkers for the diagnosis, treatment, and prognosis of cervical cancer, but further work is needed to elucidate the detailed mechanisms of IncRNAs.

Until now, only several ceRNAs related to cervical cancer have been revealed (Liu Q. et al., 2017; Shen et al., 2017; Yang et al., 2017), and the complex ceRNA network related to cervical cancer remained unclear. For the first time we systematically reported the ceRNA network based on DE IncRNAs, circRNAs, miRNAs, and mRNAs. Part of these miRNAs have been linked to cervical cancer, and they all target a number of IncRNAs, circRNAs, and mRNAs, including the majority of the novel identified non-coding RNAs; this presents the functional complexity of mRNAs and non-coding RNAs, and also indicates that these novel lncRNAs and circRNAs may be involved in cervical cancer and worthy of further investigation. There are some limitations in our study. Some elaborate experiments, such as overexpressing IncRNAs, need to be performed to elucidate the molecular mechanisms underlying the roles of non-coding RNAs. There is the possibility that some of the differences in RNA expression found in this study might not reflect the exact differences between normal cells and cancer cells because the cancer tissues contained not only cancer cells but also surrounding mesenchymal cells as well as inflammatory cells. Our future work will focus on further validating the miRNA-mediated ceRNA network and exploring the role of this network in the progression and metastasis of cervical cancer.

\section{CONCLUSION}

This study presents a profile of DE coding and non-coding RNAs, which include 3 novel lncRNAs and 44 novel circRNAs. A series of integrated analysis found that DE transcripts, including the majority of the novel identified non-coding RNAs, may be associated with the tumorigenesis and development of cervical cancer. DE non-coding RNAs could be further developed as prospective biomarkers. This study broadens our understanding of non-coding RNAs participating in the occurrence, development, and prognosis of cervical cancer, and 
it will pave the way to develop novel clinical diagnostics and therapeutic approaches.

\section{AVAILABILITY OF DATA}

The raw reads from RNA-seq are available in the National Center for Biotechnology Information (NCBI) Sequence Read Archive (SRA) (accession number: SRP114925). The authors declare that all other data are available in the article and its Supplementary Materials or from the corresponding author on reasonable request.

\section{ETHICS STATEMENT}

This study was carried out in accordance with the recommendations of "ethics committee of Union Hospital" with written informed consent from all subjects. All subjects gave written informed consent in accordance with the Declaration of Helsinki. The protocol was approved by the "Union Hospital, Tongji Medical College.”

\section{AUTHOR CONTRIBUTIONS}

HW, YZ, and JC conceived and designed the experiments. HW, $\mathrm{YZ}, \mathrm{MC}$, and JC performed the experiments. MC and JC analyzed

\section{REFERENCES}

Cao, J. (2014). The functional role of long non-coding RNAs and epigenetics. Biol. Proced. Online 16:11. doi: 10.1186/1480-9222-16-11

Chen, J., Yao, D., Zhao, S., He, C., Ding, N., Li, L., et al. (2014). MiR-1246 promotes $\mathrm{SiHa}$ cervical cancer cell proliferation, invasion, and migration through suppression of its target gene thrombospondin 2. Arch. Gynecol. Obstet. 290, 725-732. doi: 10.1007/s00404-014-3260-2

de Freitas, A. C., Coimbra, E. C., and Leitao Mda, C. (2014). Molecular targets of HPV oncoproteins: potential biomarkers for cervical carcinogenesis. Biochim. Biophys. Acta 1845, 91-103. doi: 10.1016/j.bbcan.2013.12.004

de Sanjose, S., Quint, W. G., Alemany, L., Geraets, D. T., Klaustermeier, J. E., Lloveras, B., et al. (2010). Human papillomavirus genotype attribution in invasive cervical cancer: a retrospective cross-sectional worldwide study. Lancet Oncol. 11, 1048-1056. doi: 10.1016/s1470-2045(10)70230-8

Dong, Y., He, D., Peng, Z., Peng, W., Shi, W., Wang, J., et al. (2017). Circular RNAs in cancer: an emerging key player. J. Hematol. Oncol. 10:2. doi: 10.1186/s13045016-0370-2

Fitzmaurice, C., Allen, C., Barber, R. M., Barregard, L., Bhutta, Z. A., Brenner, H., et al. (2017). Global, regional, and national cancer incidence, mortality, years of life lost, years lived with disability, and disability-adjusted life-years for 32 cancer groups, 1990 to 2015: a systematic analysis for the global burden of disease study. JAMA Oncol. 3, 524-548. doi: 10.1001/jamaoncol.2016. 5688

Hansen, T. B., Jensen, T. I., Clausen, B. H., Bramsen, J. B., Finsen, B., Damgaard, C. K., et al. (2013). Natural RNA circles function as efficient microRNA sponges. Nature 495, 384-388. doi: 10.1038/nature11993

Hauptman, N., and Glavac, D. (2013). Long non-coding RNA in cancer. Int. J. Mol. Sci. 14, 4655-4669. doi: 10.3390/ijms14034655

Huang da, W., Sherman, B. T., and Lempicki, R. A. (2009). Systematic and integrative analysis of large gene lists using DAVID bioinformatics resources. Nat. Protoc. 4, 44-57. doi: 10.1038/nprot.2008.211 the data. YZ and JC contributed reagents/materials/analysis tools. $\mathrm{MC}$ and JC wrote the paper.

\section{ACKNOWLEDGMENTS}

JC is supported by the CAS Pioneer Hundred Talents Program and $\mathrm{YZ}$ is supported by the National Natural Science Foundation of China (Grant No. 81502657).

\section{SUPPLEMENTARY MATERIAL}

The Supplementary Material for this article can be found online at: http://journal.frontiersin.org/article/10.3389/fmicb. 2017.01720/full\#supplementary-material

FIGURE S1 | KEGG pathway analyses of DE coding and non-coding RNAs. KEGG pathway enrichment analysis of DE IncRNAs (A), miRNAs (B), and mRNAs (C) with a top-ten enrichment score. MiRNAs with less than 10 pathways were enriched.

FIGURE S2 | The ceRNA network, including circRNAs, miRNAs, and mRNAs, in cervical cancer. The ceRNA network is based on circRNA/miRNA and miRNA/mRNA interactions. The size of each node is proportional to the calculated functional connectivity of each node.

FIGURE S3 | The ceRNA network, including IncRNAs, circRNAs, miRNAs, and mRNAs, in cervical cancer. The ceRNA network is based on IncRNA/miRNA, circRNA/miRNA, and miRNA/mRNA interactions. The size of each node is proportional to the calculated functional connectivity of each node.

Insinga, R. P., Perez, G., Wheeler, C. M., Koutsky, L. A., Garland, S. M., Leodolter, S., et al. (2011). Incident cervical HPV infections in young women: transition probabilities for CIN and infection clearance. Cancer Epidemiol. Biomarkers Prev. 20, 287-296. doi: 10.1158/1055-9965.epi-10-0791

Langmead, B., Trapnell, C., Pop, M., and Salzberg, S. L. (2009). Ultrafast and memory-efficient alignment of short DNA sequences to the human genome. Genome Biol. 10:R25. doi: 10.1186/gb-2009-10-3-r25

Lee, J., Yoon, Y. S., and Chung, J. H. (2008). Epigenetic silencing of the WNT antagonist DICKKOPF-1 in cervical cancer cell lines. Gynecol. Oncol. 109, 270-274. doi: 10.1016/j.ygyno.2008.01.034

Lee, J. J., Kim, M., and Kim, H. P. (2016). Epigenetic regulation of long noncoding RNA UCA1 by SATB1 in breast cancer. BMB Rep. 49, 578-583.

Li, J., Hu, L., Tian, C., Lu, F., Wu, J., and Liu, L. (2015). microRNA-150 promotes cervical cancer cell growth and survival by targeting FOXO4. BMC Mol. Biol. 16:24. doi: 10.1186/s12867-015-0052-6

Li, L. J., Zhao, W., Tao, S. S., Leng, R. X., Fan, Y. G., Pan, H. F., et al. (2017). Competitive endogenous RNA network: potential implication for systemic lupus erythematosus. Expert Opin. Ther. Targets 21, 639-648. doi: 10.1080/ 14728222.2017.1319938

Ling, S., Ruiqin, M., Guohong, Z., Bing, S., and Yanshan, C. (2015). Decreased microRNA-206 and its function in cervical cancer. Eur. J. Gynaecol. Oncol. 36, $716-721$.

Liu, J. T., Guo, W. B., and Sun, J. Y. (2017). Serum Dickkopf-1 acts as a new biomarker in human breast cancer. Minerva Med. 108, 334-340. doi: 10.23736/ s0026-4806.17.04807-8

Liu, Q., Guo, X., Que, S., Yang, X., Fan, H., Liu, M., et al. (2017). LncRNA RSU1P2 contributes to tumorigenesis by acting as a ceRNA against let-7a in cervical cancer cells. Oncotarget 8, 43768-43781. doi: 10.18632/oncotarget.10844

Louie, K. S., Castellsague, X., de Sanjose, S., Herrero, R., Meijer, C. J., Shah, K., et al. (2011). Smoking and passive smoking in cervical cancer risk: pooled analysis of couples from the IARC multicentric case-control studies. Cancer Epidemiol. Biomarkers Prev. 20, 1379-1390. doi: 10.1158/1055-9965.epi-11-0284 
Love, M. I., Huber, W., and Anders, S. (2014). Moderated estimation of fold change and dispersion for RNA-seq data with DESeq2. Genome Biol. 15, 550. doi: 10.1186/s13059-014-0550-8

Luo, M., Shen, D., Zhou, X., Chen, X., and Wang, W. (2013). MicroRNA-497 is a potential prognostic marker in human cervical cancer and functions as a tumor suppressor by targeting the insulin-like growth factor 1 receptor. Surgery 153, 836-847. doi: 10.1016/j.surg.2012.12.004

McLaughlin-Drubin, M. E., Meyers, J., and Munger, K. (2012). Cancer associated human papillomaviruses. Curr. Opin. Virol. 2, 459-466. doi: 10.1016/j.coviro. 2012.05.004

Meng, X., Li, X., Zhang, P., Wang, J., Zhou, Y., and Chen, M. (2016). Circular RNA: an emerging key player in RNA world. Brief. Bioinform. 18, 547-557. doi: 10.1093/bib/bbw045

Nie, W., Ge, H. J., Yang, X. Q., Sun, X., Huang, H., Tao, X., et al. (2016). LncRNAUCA1 exerts oncogenic functions in non-small cell lung cancer by targeting miR-193a-3p. Cancer Lett. 371, 99-106. doi: 10.1016/j.canlet.2015.11.024

Okegawa, T., Pong, R. C., Li, Y., and Hsieh, J. T. (2004). The role of cell adhesion molecule in cancer progression and its application in cancer therapy. Acta Biochim. Pol. 51, 445-457.

Orom, U. A., Derrien, T., Beringer, M., Gumireddy, K., Gardini, A., Bussotti, G., et al. (2010). Long noncoding RNAs with enhancer-like function in human cells. Cell 143, 46-58. doi: 10.1016/j.cell.2010.09.001

Ponjavic, J., Oliver, P. L., Lunter, G., and Ponting, C. P. (2009). Genomic and transcriptional co-localization of protein-coding and long non-coding RNA pairs in the developing brain. PLOS Genet. 5:e1000617. doi: 10.1371/journal. pgen. 1000617

Ponting, C. P., Oliver, P. L., and Reik, W. (2009). Evolution and functions of long noncoding RNAs. Cell 136, 629-641. doi: 10.1016/j.cell.2009.02.006

Qu, S., Yang, X., Li, X., Wang, J., Gao, Y., Shang, R., et al. (2015). Circular RNA: a new star of noncoding RNAs. Cancer Lett. 365, 141-148. doi: 10.1016/j.canlet. 2015.06.003

Salmena, L., Poliseno, L., Tay, Y., Kats, L., and Pandolfi, P. P. (2011). A ceRNA hypothesis: the rosetta stone of a hidden RNA language? Cell 146, 353-358. doi: 10.1016/j.cell.2011.07.014

Salzman, J. (2016). Circular RNA expression: its potential regulation and function. Trends Genet. 32, 309-316. doi: 10.1016/j.tig.2016.03.002

Schelhorn, S. E., Fischer, M., Tolosi, L., Altmuller, J., Nurnberg, P., Pfister, H., et al. (2013). Sensitive detection of viral transcripts in human tumor transcriptomes. PLOS Comput. Biol. 9:e1003228. doi: 10.1371/journal.pcbi.1003228

Shang, C., Guo, Y., Zhang, J., and Huang, B. (2016). Silence of long noncoding RNA UCA1 inhibits malignant proliferation and chemotherapy resistance to adriamycin in gastric cancer. Cancer Chemother. Pharmacol. 77, 1061-1067. doi: 10.1007/s00280-016-3029-3

Shannon, P., Markiel, A., Ozier, O., Baliga, N. S., Wang, J. T., Ramage, D., et al. (2003). Cytoscape: a software environment for integrated models of biomolecular interaction networks. Genome Res. 13, 2498-2504. doi: 10.1101/ gr.1239303

Shen, C. J., Cheng, Y. M., and Wang, C. L. (2017). LncRNA PVT1 epigenetically silences miR-195 and modulates EMT and chemoresistance in cervical cancer cells. J. Drug Target 25, 637-644. doi: 10.1080/1061186x.2017.1307379

Sun, J., Lin, Y., and Wu, J. (2013). Long non-coding RNA expression profiling of mouse testis during postnatal development. PLOS ONE 8:e75750. doi: 10.1371/ journal.pone.0075750

Sun, L., Jiang, R., Li, J., Wang, B., Ma, C., Lv, Y., et al. (2017). MicoRNA-425-5p is a potential prognostic biomarker for cervical cancer. Ann. Clin. Biochem. 54, 127-133. doi: 10.1177/0004563216649377

Teicher, B. A., and Fricker, S. P. (2010). CXCL12 (SDF-1)/CXCR4 pathway in cancer. Clin. Cancer Res. 16, 2927-2931. doi: 10.1158/1078-0432.ccr-092329

Trapnell, C., Roberts, A., Goff, L., Pertea, G., Kim, D., Kelley, D. R., et al. (2012). Differential gene and transcript expression analysis of RNA-seq experiments with TopHat and Cufflinks. Nat. Protoc. 7, 562-578. doi: 10.1038/nprot. 2012.016

Trapnell, C., Williams, B. A., Pertea, G., Mortazavi, A., Kwan, G., van Baren, M. J., et al. (2010). Transcript assembly and quantification by RNA-Seq reveals unannotated transcripts and isoform switching during cell differentiation. Nat. Biotechnol. 28, 511-515. doi: 10.1038/nbt.1621

Vaccarella, S., Herrero, R., Dai, M., Snijders, P. J., Meijer, C. J., Thomas, J. O., et al. (2006). Reproductive factors, oral contraceptive use, and human papillomavirus infection: pooled analysis of the IARC HPV prevalence surveys. Cancer Epidemiol. Biomarkers Prev. 15, 2148-2153. doi: 10.1158/1055-9965.epi-060556

Wang, B., Huang, Z., Gao, R., Zeng, Z., Yang, W., Sun, Y., et al. (2017). Expression of long noncoding RNA urothelial cancer associated 1 promotes cisplatin resistance in cervical cancer. Cancer Biother. Radiopharm. 32, 101-110. doi: $10.1089 /$ cbr.2016.2156

Wang, F., Nazarali, A. J., and Ji, S. (2016). Circular RNAs as potential biomarkers for cancer diagnosis and therapy. Am. J. Cancer Res. 6, 1167-1176.

Wang, L., Feng, Z., Wang, X., Wang, X., and Zhang, X. (2010). DEGseq: an $\mathrm{R}$ package for identifying differentially expressed genes from RNA-seq data. Bioinformatics 26, 136-138. doi: 10.1093/bioinformatics/btp612

Xie, C., Mao, X., Huang, J., Ding, Y., Wu, J., Dong, S., et al. (2011). KOBAS 2.0: a web server for annotation and identification of enriched pathways and diseases. Nucleic Acids Res. 39, W316-W322. doi: 10.1093/nar/ gkr483

Xiong, Y., Huang, F., Li, X., Chen, Z., Feng, D., Jiang, H., et al. (2017). CCL21/CCR7 interaction promotes cellular migration and invasion via modulation of the MEK/ERK1/2 signaling pathway and correlates with lymphatic metastatic spread and poor prognosis in urinary bladder cancer. Int. J. Oncol. 51, 75-90. doi: 10.3892/ijo.2017.4003

Xu, J., Lange, E. M., Lu, L., Zheng, S. L., Wang, Z., Thibodeau, S. N., et al. (2013). HOXB13 is a susceptibility gene for prostate cancer: results from the International Consortium for Prostate Cancer Genetics (ICPCG). Hum. Genet. 132, 5-14. doi: 10.1007/s00439-012-1229-4

Xu, Y., Zhao, S., Cui, M., and Wang, Q. (2015). Down-regulation of microRNA$135 \mathrm{~b}$ inhibited growth of cervical cancer cells by targeting FOXO1. Int. J. Clin. Exp. Pathol. 8, 10294-10304.

Yang, W., Hong, L., Xu, X., Wang, Q., Huang, J., and Jiang, L. (2017). LncRNA GAS5 suppresses the tumorigenesis of cervical cancer by downregulating miR-196a and miR-205. Tumour Biol. 39:1010428317711315. doi: 10.1177/ 1010428317711315

Ye, H., Yu, X., Xia, J., Tang, X., Tang, L., and Chen, F. (2016). MiR-486-3p targeting ECM1 represses cell proliferation and metastasis in cervical cancer. Biomed. Pharmacother. 80, 109-114. doi: 10.1016/j.biopha.2016.02.019

Zhang, X., Wang, Y., Cao, Y., Zhang, X., and Zhao, H. (2017). Increased CCL19 expression is associated with progression in cervical cancer. Oncotarget doi: 10.18632/oncotarget.17982

Zhou, L., Chen, J., Li, Z., Li, X., Hu, X., Huang, Y., et al. (2010). Integrated profiling of microRNAs and mRNAs: microRNAs located on Xq27.3 associate with clear cell renal cell carcinoma. PLOS ONE 5:e15224. doi: 10.1371/journal. pone.0015224

Conflict of Interest Statement: The authors declare that the research was conducted in the absence of any commercial or financial relationships that could be construed as a potential conflict of interest.

Copyright (c) 2017 Wang, Zhao, Chen and Cui. This is an open-access article distributed under the terms of the Creative Commons Attribution License (CC BY). The use, distribution or reproduction in other forums is permitted, provided the original author(s) or licensor are credited and that the original publication in this journal is cited, in accordance with accepted academic practice. No use, distribution or reproduction is permitted which does not comply with these terms. 